\title{
Can Tongue Acupuncture Enhance Body Acupuncture? First Results from Heart Rate Variability and Clinical Scores in Patients with Depression
}

\author{
Xian Shi, ${ }^{1}$ Huan Wang, ${ }^{1}$ Lu Wang, ${ }^{1,2}$ Zengkai Zhao, ${ }^{1}$ Daniela Litscher, ${ }^{1,2}$ Jingqiao Tao, \\ Ingrid Gaischek, ${ }^{2}$ Zemin Sheng, ${ }^{3}$ and Gerhard Litscher ${ }^{1,2}$ \\ ${ }^{1}$ Department of Acupuncture, People's Liberation Army General Hospital, Beijing 100853, China \\ ${ }^{2}$ Research Unit for Complementary and Integrative Laser Medicine, Research Unit of Biomedical Engineering in Anesthesia and \\ Intensive Care Medicine, and TCM Research Center Graz, Medical University of Graz, Auenbruggerplatz 29, 8036 Graz, Austria \\ ${ }^{3}$ Private Clinic Laßnitzhöhe, 8301 Laßnitzhöhe, Austria
}

Correspondence should be addressed to Xian Shi; 301sx@live.cn and Gerhard Litscher; gerhard.litscher@medunigraz.at

Received 4 February 2014; Revised 17 February 2014; Accepted 25 February 2014; Published 23 March 2014

Academic Editor: Cun-Zhi Liu

Copyright (C) 2014 Xian Shi et al. This is an open access article distributed under the Creative Commons Attribution License, which permits unrestricted use, distribution, and reproduction in any medium, provided the original work is properly cited.

\begin{abstract}
Tongue acupuncture (TA) is a method which is not used in western medicine and even in China it is applied very rarely in clinical practice. This study aimed at investigating whether additional TA can improve the efficacy of body acupuncture (BA) in patients with depression. Twenty patients with a mean age of \pm SD of $42.9 \pm 11.2$ years were randomly divided into two groups $(n=10$ patients each), one group receiving BA (Zusanli, Sanyinjiao, Neiguan, Shenting, Yintang, and Baihui) and the other receiving BA and TA (Liver, Heart, and Brain). The quantitative and qualitative outcome measures were heart rate (HR), heart rate variability (HRV), and different clinical scores. We found that in both groups all scores and HR improved significantly, whereas HRV increased partly significantly. It seems that TA can enhance acute and treatment effects of BA in patients with depression. The investigation of de qi sensation in TA needs further attention.
\end{abstract}

\section{Introduction}

Tongue acupuncture (TA) is a unique natural therapy. Specific tongue acupoints are supposed to be related to various functional domains of the body. It is claimed that TA can stimulate different meridians associated with different organs' functions in order to adjust blood circulation and energyflow in the body [1].

De qi sensation evoked by TA is essential to its clinical effectiveness. One purpose of TA is restoring consciousness and brain resuscitation. In China, it is used as a complementary method to treat not only several diseases, like stroke [2], but also children with visual impairment [3].

In contrast to TA, there are a lot of clinical investigations concerning body acupuncture (BA) and major depressive disorders [4].

Figures 1(a) and 1(b) summarize the number of publications concerning different terms related to TA and BA.
Our research group recently found acute stimulation effects on neurovegetative parameters like heart rate (HR) and heart rate variability (HRV) in patients with depression [5] and insomnia [6].

The present clinical study was performed at the Military Acupuncture Centre at the People's Liberation Army General Hospital, Beijing, in cooperation with the Traditional Chinese Medicine (TCM) Research Center Graz, Austria (http://litscher.info/ and http://tcm-graz.at/). This paper compares results from BA and BA + TA measurements in depression patients with regard to neurophysiological parameters like HR and HRV.

\section{Materials and Methods}

2.1. Patients. Twenty patients (all females; mean age \pm SD $42.9 \pm 11.2$ years; range $20-65$ years) suffering from depression (Chinese diagnosis "Yu Zheng") received acupuncture 


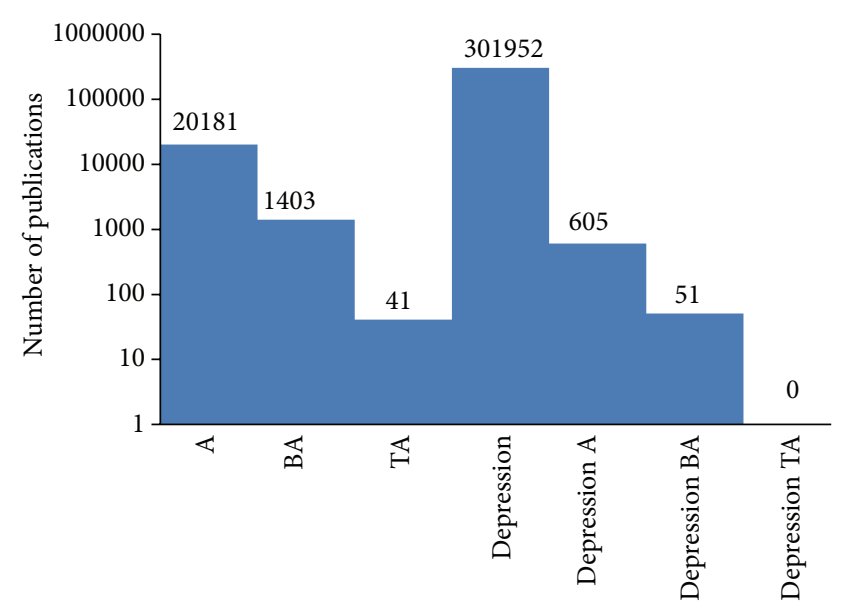

(a)

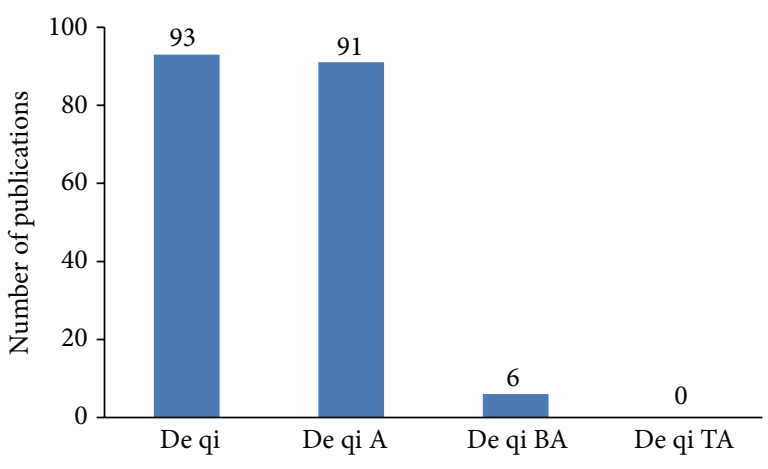

(b)

FIGURE 1: Use of different acupuncture approaches (http://www.pubmed.gov/): A ... acupuncture; BA ... body acupuncture; and TA ... tongue acupuncture.

treatment at the Chinese People's Liberation Army Hospital, Beijing. The clinical evaluation of the patients, performed before the first and after the last acupuncture session, used three main scales: the Hamilton Anxiety Rating Scale (HAMA) [7], the Athens Insomnia Scale (AIS) [8], and the Hamilton Rating Scale for Depression (HRSD) [9]. No patient was under the influence of centrally active medication or had a history of heart or cerebrovascular disease, respiratory or neurological problems, or hypertension. The study was approved by the Ethics Committee of the Chinese People's Liberation Army Hospital and carried out in compliance with the Declaration of Helsinki. All patients gave oral informed consent.

2.2. Teleacupuncture. Electrocardiographic (ECG) biosignal recording was performed in China, and data analysis took place in Europe. For ECG registration three adhesive electrodes (Skintact Premier F-55, Leonhard Lang GmbH, Innsbruck, Austria) were used which were applied to the chest.

The research team in China used a medilog AR12 HRV (Huntleigh Healthcare, Cardiff, United Kingdom) system from the TCM Research Center at the Medical University in Graz. The system has a sampling rate of $4096 \mathrm{~Hz}$; the raw data are stored digitally and transferred to the TCM Research Center Graz via the Internet. The biosignals were analyzed and HRV was calculated.

Like in previous studies $[5,6,10]$, mean HR, total HRV, and the LF (low frequency)/HF (high frequency) ratio of HRV were chosen as evaluation parameters, as such being recommended by the Task Force of the European Society of Cardiology and the North American Society of Pacing and Electrophysiology [11].

\subsection{Body Acupuncture, Tongue Acupuncture, and Procedure.} The patients were randomly divided into two groups using a simple randomization (numbers by chance). One group ( $n=10$; mean age $39.2 \pm 13.2$ years; range $20-65$ years)

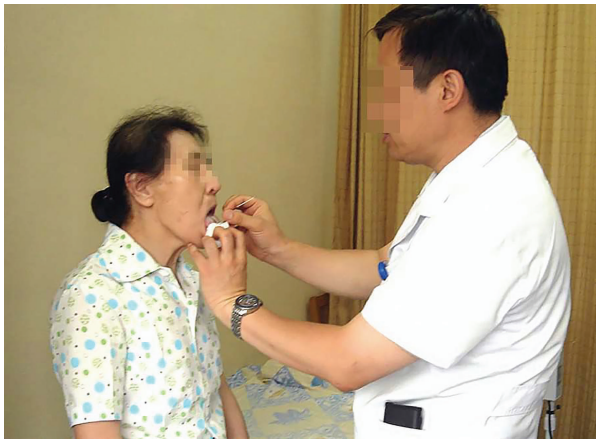

Figure 2: Tongue acupuncture at the Military Acupuncture Training Centre at the People's Liberation Army General Hospital, Beijing.

received BA in six sessions and the other group $(n=10$; mean age $46.6 \pm 7.8$ years; range $38-60$ years) additionally received TA (see Figure 2) in all six sessions. HR-HRV recordings were performed during the first and the last acupuncture session.

The following body acupoints were used in this study: Zusanli (ST36,bilateral), Sanyinjiao (SP6, bilateral), Neiguan (PC6, bilateral), Shenting (GV24), Yintang (Ex-HN3), and Baihui (GV20). The sterile, single-use needles $(0.30 \times 25 \mathrm{~mm}$, Huan Qiu, Suzhou, China) were inserted perpendicularly, and the needle was stimulated clockwise and counterclockwise for 15 seconds each, with six rotations per second, resulting in 90 rotations per stimulation.

The patients in the TA group received TA in every acupuncture session, immediately before BA. For TA, the points Liver, Heart, and Brain (Figure 3) were always used in this order [1]. The needle was inserted obliquely into the tongue acupuncture point, to a depth of $0.5-1$ cun, and immediately removed again; then the next tongue point was treated likewise.

The measurement profile and measurement phases (ad) of the BA treatment are shown in Figure 4. Four registration periods ( $5 \mathrm{~min}$ each) were compared: one before 

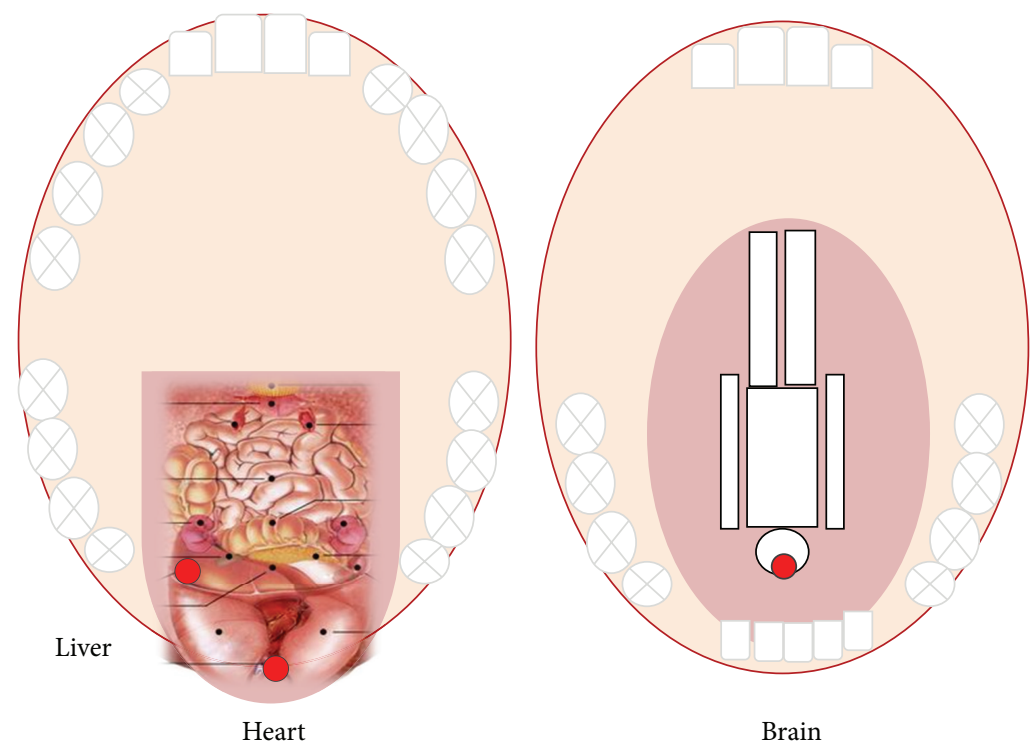

FIGURE 3: Tongue acupuncture points used in this study.

TABLE 1: Changes in clinical scores and blood pressure values between the first (M1) and last (M2) treatment session. Values are given as mean \pm SD (standard deviation).

\begin{tabular}{lccccc}
\hline & & Body acupuncture & & \multicolumn{3}{c}{ Body + tongue acupuncture } \\
& M1 & M2 & $P$ & M1 & M2 \\
\hline HAM-A & $18.9 \pm 3.9$ & $16.6 \pm 3.5$ & 0.008 & $24.4 \pm 7.9$ & $19.0 \pm 7.1$ \\
AIS & $15.0 \pm 6.7$ & $11.3 \pm 5.1$ & $<0.001$ & $13.9 \pm 4.0$ & $8.3 \pm 3.2$ \\
HRSD & $22.2 \pm 5.4$ & $19.5 \pm 4.8$ & 0.001 & $22.3 \pm 5.9$ & $17.4 \pm 4.8$ \\
BPsys [mmHg] & $105.3 \pm 9.8$ & $103.6 \pm 9.8$ & n.s. & $113.3 \pm 10.4$ & $113.1 \pm 7.4$ \\
BPdia [mmHg] & $66.4 \pm 4.3$ & $66.3 \pm 4.7$ & n.s. & $69.2 \pm 6.4$ & 6.001 \\
\hline
\end{tabular}

HAM-A: Hamilton Anxiety Rating Scale [7]; AIS: Athens Insomnia Scale [8]; HRSD: Hamilton Rating Scale for Depression [9]; BPsys: systolic blood pressure; BPdia: diastolic blood pressure; and n.s.: not significant.

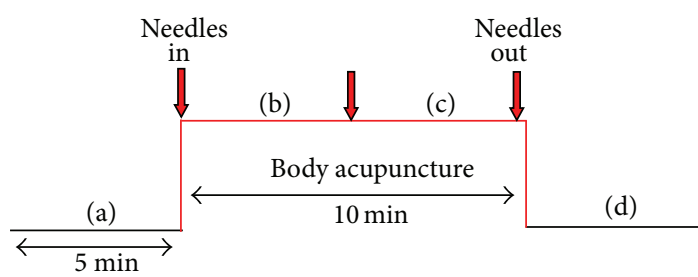

FIgURE 4: Measurement procedure for body acupuncture.

acupuncture (a), two during acupuncture $(b, c)$, and one as a control after removing the needles (d). In addition, blood pressure was measured at the beginning and at the end of the acupuncture sessions.

2.4. Statistical Analysis. Data were analyzed using SigmaPlot 12.0 software (Systat Software Inc., Chicago, USA). Graphical presentation of results uses box plot illustrations. Testing was performed with Friedman repeated measures ANOVA on ranks and Tukey or Holm-Sidak test. The criterion for significance was $P<0.05$.

\section{Results}

Figures 5 and 6 show the mean HR and total HRV from the ECG recordings of both patient groups during the first and last treatment session, respectively. HR had decreased significantly in both groups in the course of the treatment.

In contrast to HR, HRV had increased significantly in the BA group during the course of the treatment (see Figure 6).

Furthermore, continuous HRV monitoring showed significant alterations in the LF/HF ratio within the single treatment sessions (see Figure 7). The comparison between the first and the last treatment, however, did not reach the level of significance.

The direct statistical comparison between the TA and BA group did not yield significant changes; however, there was a marked decrease of HR in the TA group and the decrease of the HAM-A score (see Table 1) showed a higher significance ( $P=0.008$ in the BA group and $P<0.001$ in the TA group).

The analysis of the three clinical scores revealed interesting results. In all scores there was a significant reduction (see Table 1).

The data of the blood pressure values showed insignificant results (see Table 1). The de qi sensation during TA was 


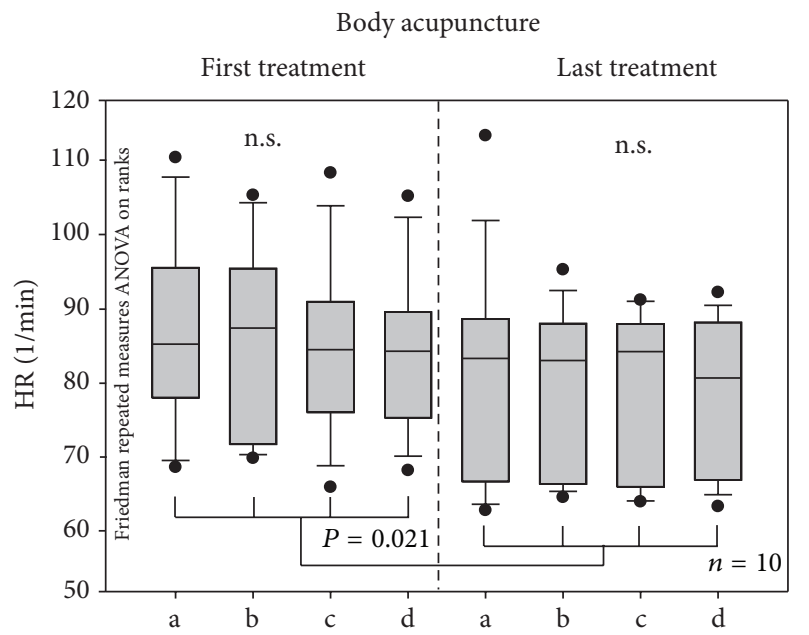

(a)
Body + tongue acupuncture

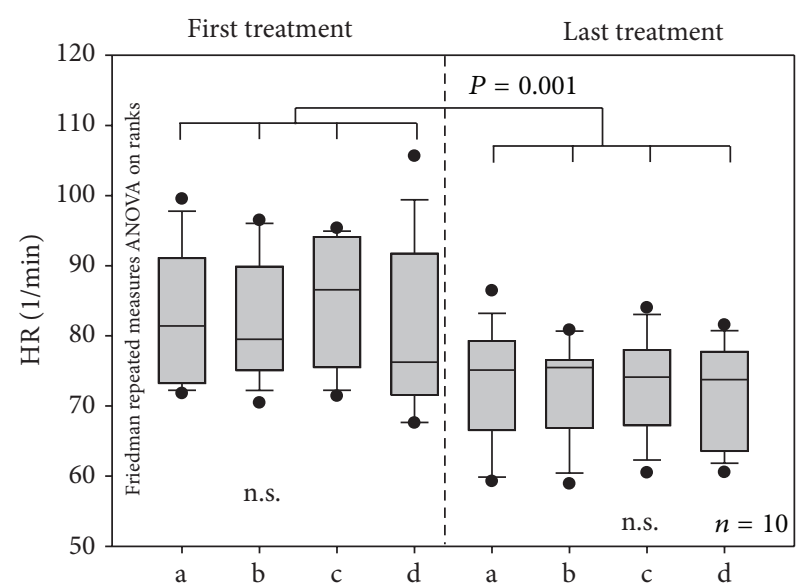

(b)

FIGURE 5: Box plots displaying the changes in mean heart rate (HR). Within the single treatment sessions, no significant effects could be found. When comparing the values of the first session to those of the last session, however, HR had decreased significantly. The ends of the boxes define the 25 th and 75 th percentiles with a line at the median and error bars defining the 10th and 90th percentiles.

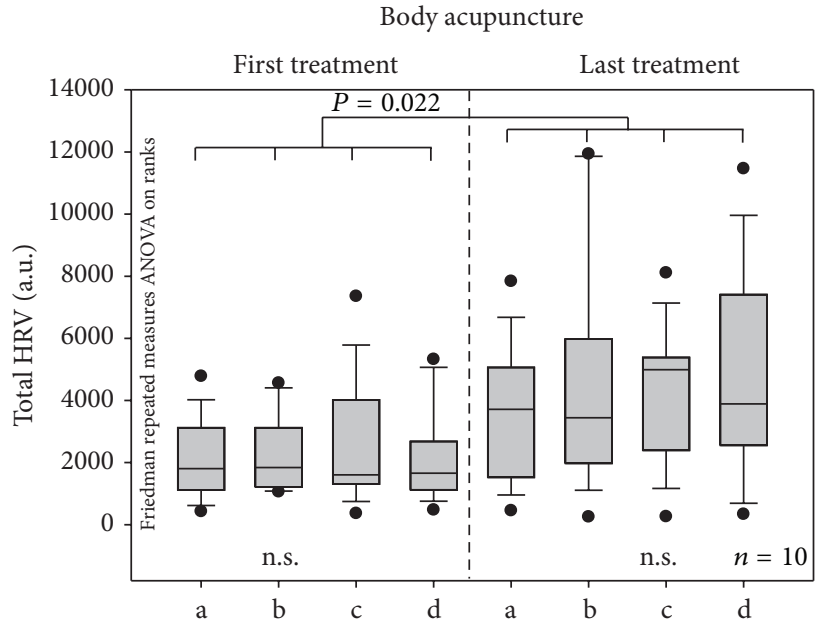

(a)

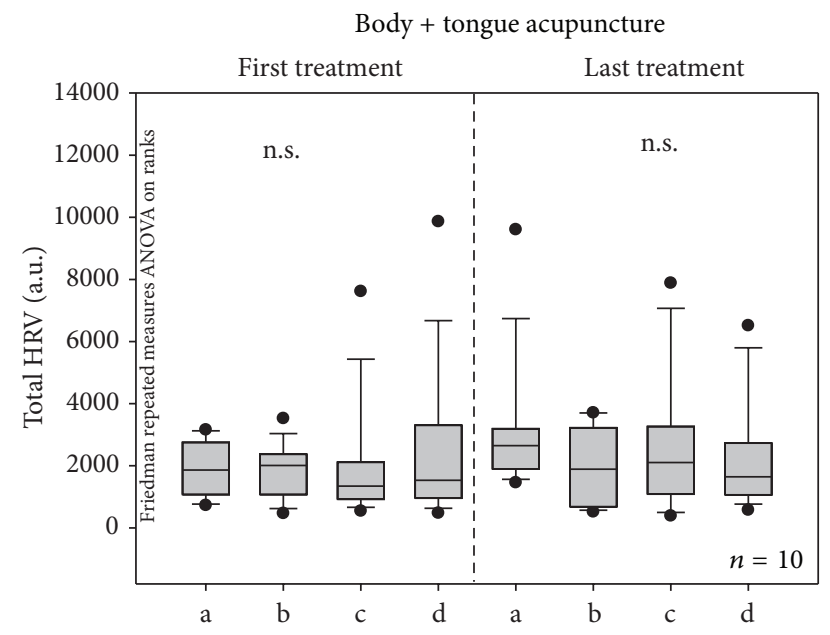

(b)

FIGURE 6: Changes in total heart rate variability (HRV). For further explanations, see Figure 5.

similar to that during BA. After the arrival of qi, the TA patients reported sensations of distension, heaviness, and numbness; in addition, these sensations spread to the throat.

\section{Discussion}

Tongue acupuncture is an innovative but not commonly used technique in the traditional Chinese medicine [12]. It originated from the theory of TCM through scientific research. Forty points on the tongue were discovered that correspond to organs and certain parts of the body [1]. Studies showed that TA and BA can improve the visual status of children with visual disorders, both peripheral and central in origin [3]. The authors of a rat model study monitored the evoked activity of the digastric electromyography elicited by electrical stimulation of the tongue [13]. In a study dealing with aphasia after stoke, authors mentioned that tongue bleeding, deep insertion, and strong stimulation were adopted by many practitioners [14]. Li et al. found that tongue acupuncture has a better therapeutic effect on stroke [2]. There are also a few other studies available in the database PubMed which also deal with the topic tongue acupuncture; however, there are no publications available in connection with depression or de qi sensation (Section 1).

In Asia and also in Europe, depression is one of the most disabling diseases, causing a significant burden both to the individual and to society [15-17]. Detailed information on this important topic can be found in the discussion section 


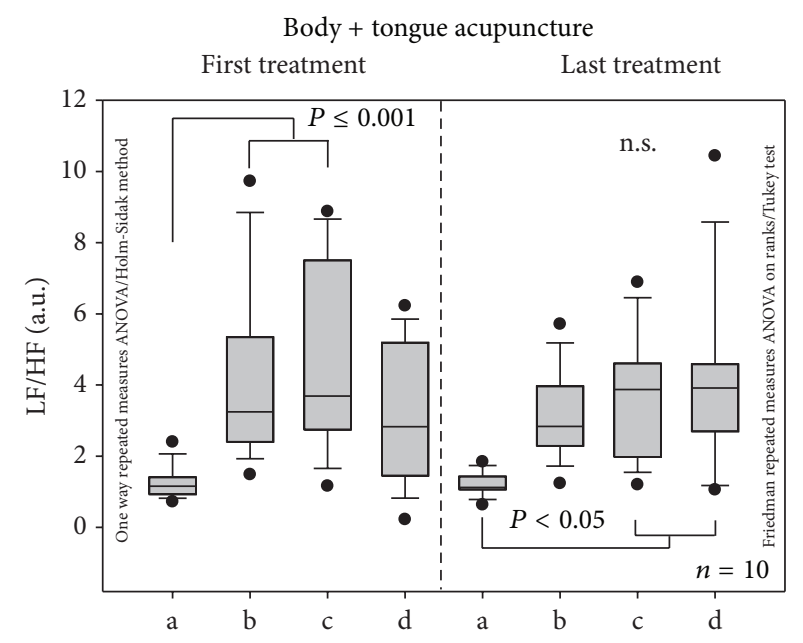

(a)

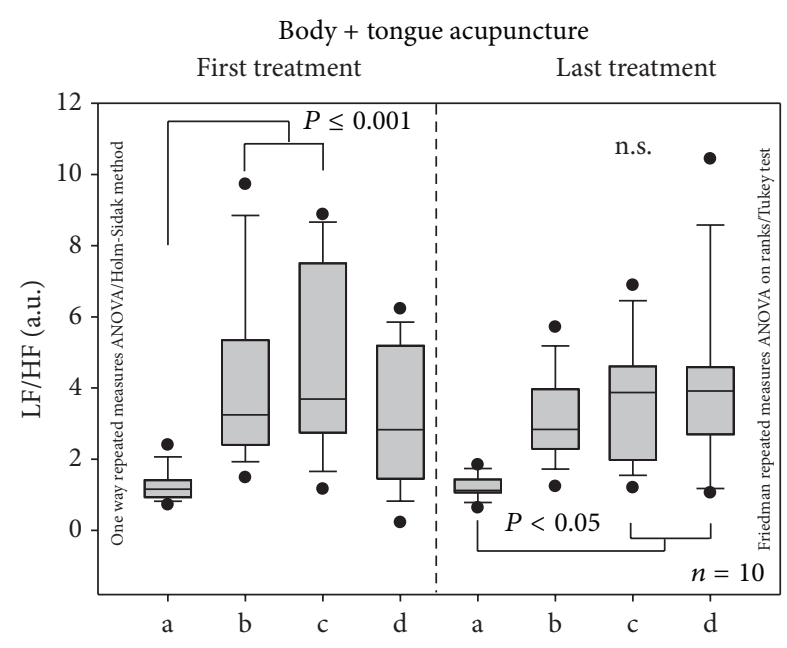

(b)

Figure 7: The low frequency (LF)/high frequency (HF) ratio did not change significantly when comparing the first and last treatment. For further explanations, see Figure 5.

of one of our previous publications published recently [15]. As stated in that article, one important way to stop this cost explosion in China and Europe is through increased research efforts in this field. Better detection, prevention, treatment, and patient management are imperative to reduce the burden of depression and its cost [15-17].

As previously described, continuous electrical auricular acupuncture is one special kind and new method to treat patients with neurological diseases like depression [15]. The results of our present study are in accordance with previous investigations [15]. All clinical scores (HAM-A, AIS, and HRSD) showed a significant improvement already after 6 TA/BA and BA sessions. However, it has to be mentioned that the baseline values of the HAM-A score differed between the two treatment groups because randomization was performed by chance and not by score assessment. In addition, HR and HRV, which are reliable indicators of the state of health $[5,6,15]$, also improved, partly significantly.

When performing TA, most acupuncturists do not leave the needle in place; they puncture the surface of the tongue and describe the fact that they receive good therapeutic effects in some clinical applications like in apoplectic aphasia rehabilitation [14]. Horizontal and deep puncturing approaches were also sometimes used. This method refers to piercing the tongue from one side to the other $[18,19]$. Deep puncturing needs long needles for treatment towards the root of the tongue [20].

In conclusion, our study shows clinical (scores) and neurovegetative (HRV) improvements in parameters after BA and TA acupoint stimulation in patients with depression. It can be stated that invasive tongue stimulation with needles does not have inhibitory effects on BA; on the contrary, it seems that it can enhance acute and treatment effects of BA. The investigation of de qi sensation [21] needs further attention. Up to now, it has not been described in detail in English or even in Chinese scientific literature.

\section{Conflict of Interests}

The authors declare that they have no conflict of interests regarding the publication of this paper.

\section{Authors' Contribution}

Xian Shi and Huan Wang contributed equally to this study.

\section{Acknowledgments}

The scientific investigations were supported by the Austrian Federal Ministries of Economy and Science and Health, the Eurasia-Pacific Uninet (project "Evidence-based high-tech acupuncture and integrative laser medicine for prevention and early intervention of chronic diseases"), and the German Academy of Acupuncture.

\section{References}

[1] J. G. Sun and X. R. Sun, “Tongue acupuncture," Zhongguo Zhen Jiu, vol. 30, no. 4, pp. 347-348, 2010.

[2] Q. Li, Z. H. Wang, J. Ye, X. Y. Zhu, and Z. H. Guan, "Clinical observation on tongue acupuncture for treatment of stroke," Zhongguo Zhen Jiu, vol. 25, no. 11, pp. 820-822, 2005.

[3] V. C. Wong, J. G. Sun, and D. W. Yeung, "Pilot study of efficacy of tongue and body acupuncture in children with visual impairment," Journal of Child Neurology, vol. 21, no. 6, pp. 463473, 2006.

[4] R. Nahas and O. Sheikh, "Complementary and alternative medicine for the treatment of major depressive disorder," Canadian Family Physician, vol. 57, no. 6, pp. 659-663, 2011.

[5] L. Wang, G. Y. Cheng, Z. M. Sheng et al., "Clinical teleacupuncture between China and Austria using heart rate variability in patients with depression," Chinese Medicine, vol. 2, pp. 71-76, 2011. 
[6] G. Litscher, G. Cheng, W. Cheng et al., "Sino-European transcontinental basic and clinical high-tech acupuncture studies. Part 2. Acute stimulation effects on heart rate and its variability in patients with insomnia," Evidence-based Complementary and Alternative Medicine, vol. 2012, Article ID 916085, 5 pages, 2012.

[7] M. Hamilton, "The assessment of anxiety states by rating," The British Journal of Medical Psychology, vol. 32, no. 1, pp. 50-55, 1959.

[8] C. R. Soldatos, D. G. Dikeos, and T. J. Paparrigopoulos, "Athens insomnia scale: validation of an instrument based on ICD-10 criteria," Journal of Psychosomatic Research, vol. 48, no. 6, pp. 555-560, 2000.

[9] M. Hamilton, "A rating scale for depression," Journal of Neurology, Neurosurgery, and Psychiatry, vol. 23, pp. 56-62, 1960.

[10] G. Litscher, "Bioengineering assessment of acupuncture Part 7. Heart rate variability," Critical Reviews in Biomedical Engineering, vol. 35, no. 3-4, pp. 183-195, 2007.

[11] Task force of the European Society of Cardiology and the North American Society of Pacing and Electrophysiology, "Heart rate variability: Standards of measurement, physiological interpretation and clinical use," European Heart Journal, vol. 17, pp. 354381, 1996.

[12] V. Wong, J. G. Sun, and W. Wong, "Traditional Chinese medicine (tongue acupuncture) in children with drooling problems," Pediatric Neurology, vol. 25, no. 1, pp. 47-54, 2001.

[13] K. Okada, M. Oshima, and K. Kawakita, "Examination of the afferent fiber responsible for the suppression of jaw-opening reflex in heat, cold, and manual acupuncture stimulation in rats," Brain Research, vol. 740, no. 1-2, pp. 201-207, 1996.

[14] Y. Sun, S. A. Xue, and Z. Zuo, "Acupuncture therapy on apoplectic aphasia rehabilitation," Journal of Traditional Chinese Medicine, vol. 32, no. 3, pp. 314-321, 2012.

[15] X. Shi, G. Litscher, H. Wang et al., "Continuous auricular electroacupuncture can significantly improve heart rate variability and clinical scores in patients with depression: first results from a transcontinental study," Evidence-Based Complementary and Alternative Medicine, vol. 2013, Article ID 894096, 6 pages, 2013.

[16] T. W. Hu, Y. He, M. Zhang, and N. Chen, "Economic costs of depression in China," Social Psychiatry and Psychiatric Epidemiology, vol. 42, no. 2, pp. 110-116, 2007.

[17] P. Sobocki, B. Jönsson, J. Angst, and C. Rehnberg, "Cost of depression in Europe," Journal of Mental Health Policy and Economics, vol. 9, no. 2, pp. 87-98, 2006.

[18] Y. Y. Yang and L. X. Han, "150 cases with aphasia after stroke treated by horizontal puncturing the tongue," Shanghai Zhen Jiu Za Zhi, vol. 15, no. 3, p. 105, 1996.

[19] C. W. Tian, "186 cases with speech disability treated by puncturing tongue," Zhongguo Zhen Jiu, vol. 12, no. 5, pp. 9-44, 1992.

[20] J. F. Dong, "100 cases with language impairment after stroke treated by SheSanZhen," Zhen Jiu Lin Chuang Za Zhi, no. 1, p. 18, 1996.

[21] C. Z. Liu, G. Litscher, F. R. Liang, J. Kong, L. P. Wang, and L. Wang, "Deqi sensation in different kinds of acupuncture," Evidence-Based Complementary and Alternative Medicine, vol. 2014, Article ID 121573, 1 page, 2014. 


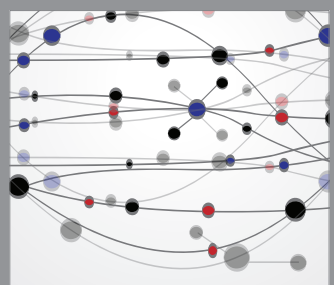

The Scientific World Journal
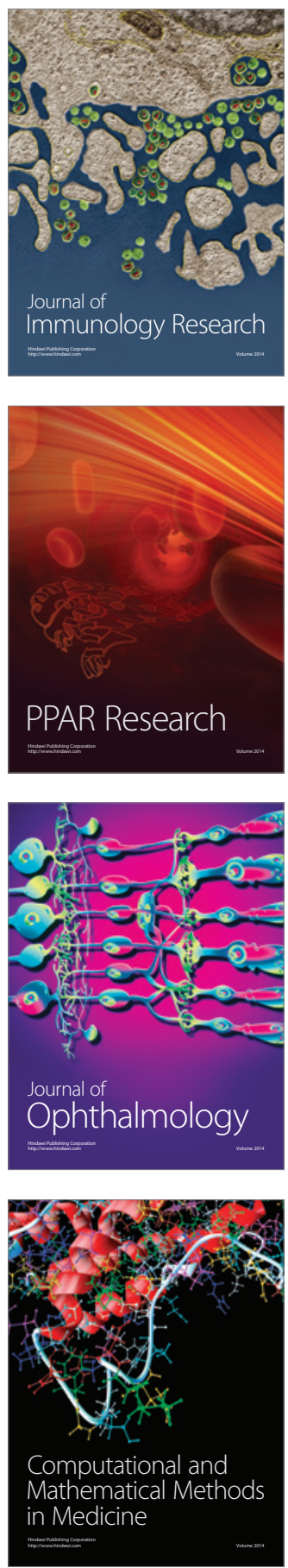

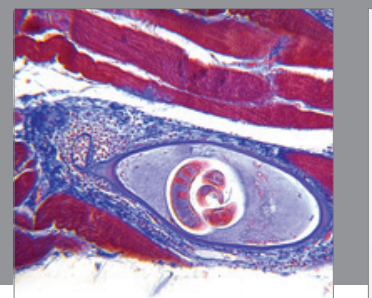

Gastroenterology

Research and Practice
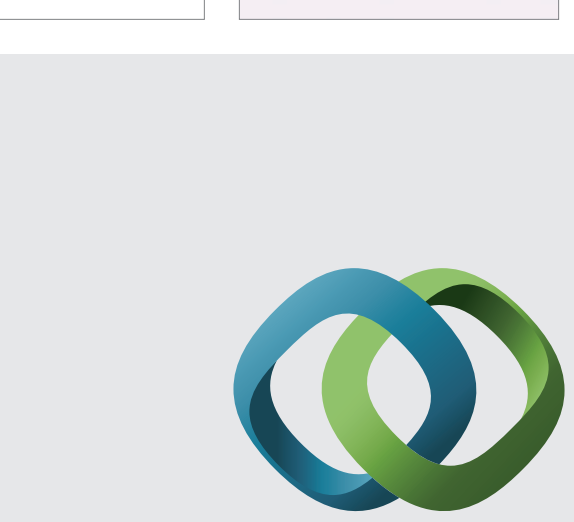

\section{Hindawi}

Submit your manuscripts at

http://www.hindawi.com
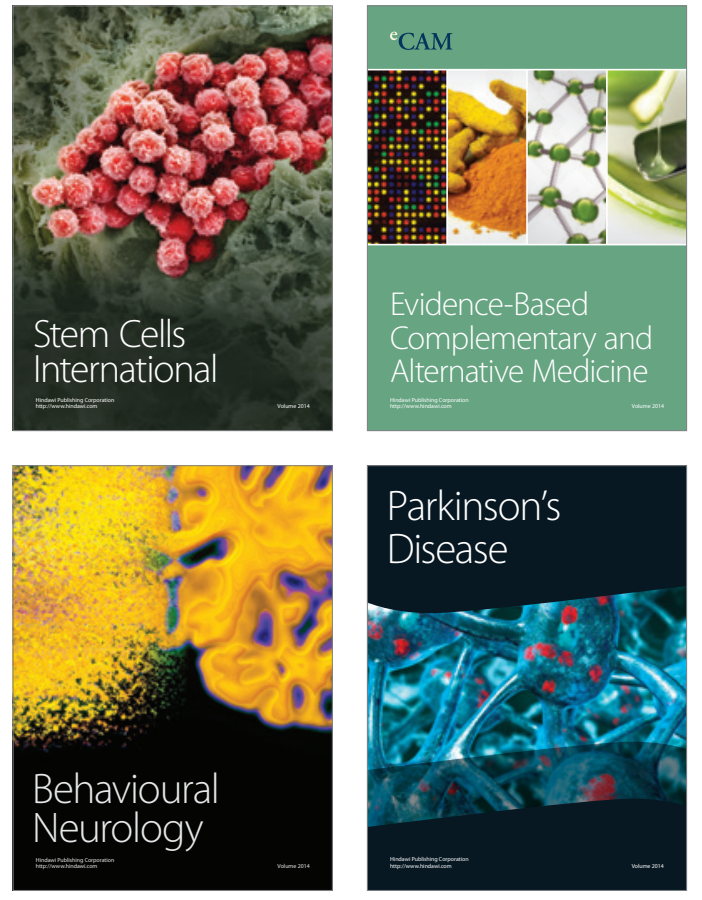
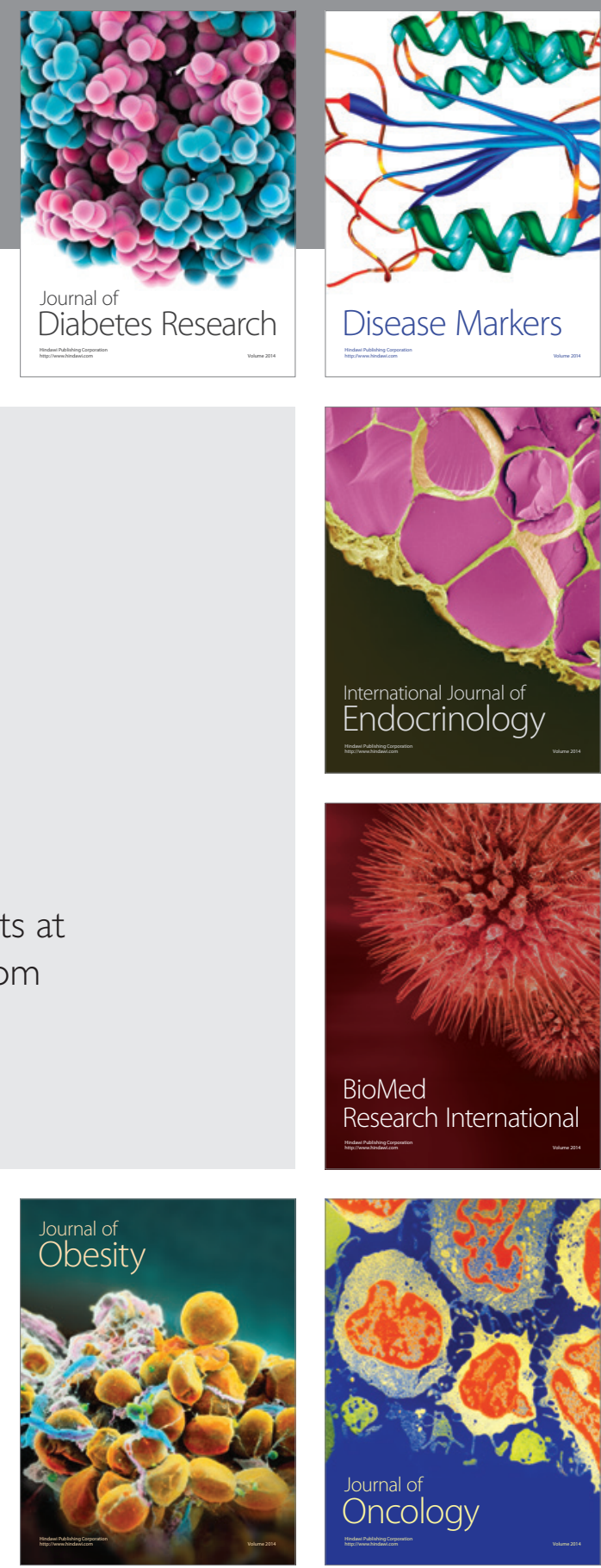

Disease Markers
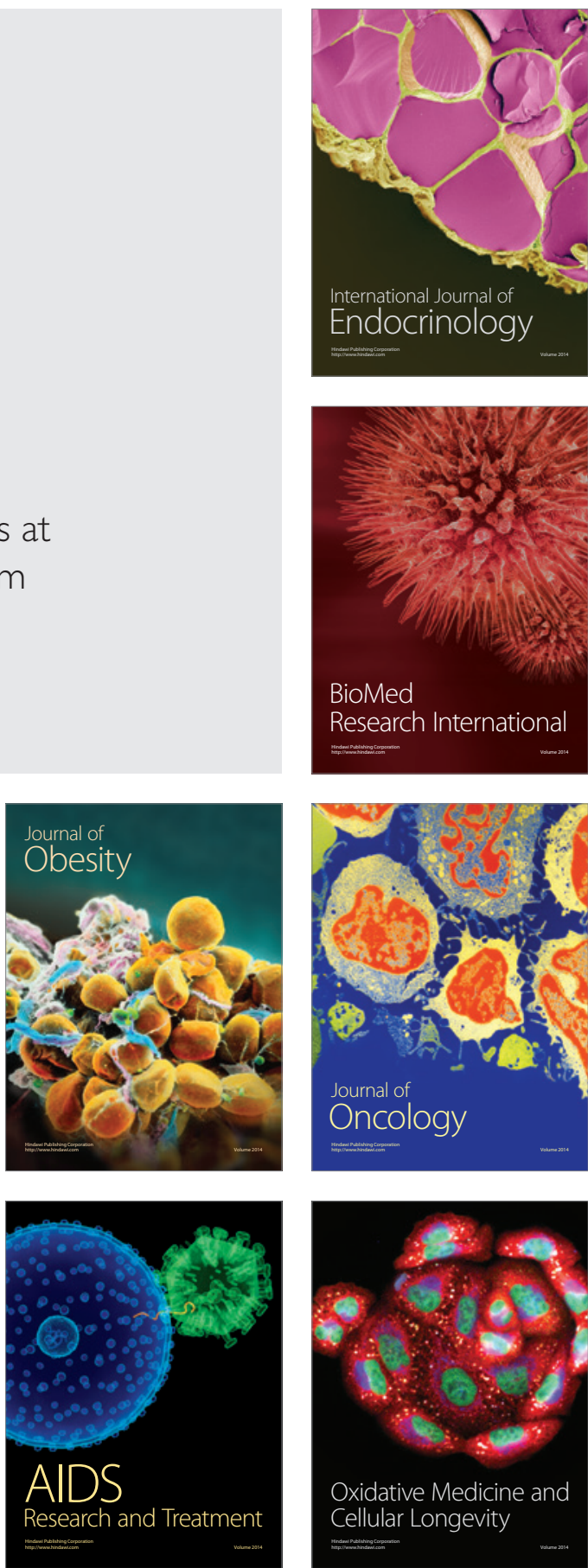